\title{
Saddle tangencies and the distance of Heegaard splittings
}

\author{
TAO LI
}

\begin{abstract}
We give another proof of a theorem of Scharlemann and Tomova and of a theorem of Hartshorn. The two theorems together say the following. Let $M$ be a compact orientable irreducible 3-manifold and $P$ a Heegaard surface of $M$. Suppose $Q$ is either an incompressible surface or a strongly irreducible Heegaard surface in $M$. Then either the Hempel distance $d(P) \leq 2 \operatorname{genus}(Q)$ or $P$ is isotopic to $Q$. This theorem can be naturally extended to bicompressible but weakly incompressible surfaces.
\end{abstract}

57N10; 57M50

\section{Introduction}

Let $P$ be a closed orientable surface of genus at least 2. The curve complex of $P$, introduced by Harvey [6], is the complex whose vertices are the isotopy classes of essential simple closed curves in $P$, and $k+1$ vertices determine a $k$-simplex if they are represented by pairwise disjoint curves. We denote the curve complex of $P$ by $\mathcal{C}(P)$. For any two vertices in $\mathcal{C}(P)$, the distance $d(x, y)$ is the minimal number of 1-simplices in a simplicial path jointing $x$ to $y$. To simplify notation, unless necessary, we do not distinguish a vertex in $\mathcal{C}(P)$ from a simple closed curve in $P$ representing this vertex.

Let $M$ be a compact orientable irreducible 3-manifold and $P$ an embedded connected separating surface in $M$ with genus $(P) \geq 2$. Let $U$ and $V$ be the closure of the two components of $M-P$. We may view $\partial U=\partial V=P$. As in Scharlemann-Tomova [14], we say $P$ is bicompressible if $P$ is compressible in both $U$ and $V$. Let $\mathcal{U}$ and $\mathcal{V}$ be the set of vertices in $\mathcal{C}(P)$ represented by curves bounding compressing disks in $U$ and $V$ respectively. The distance $d(P)$ is defined to be the distance between $\mathcal{U}$ and $\mathcal{V}$ in the curve complex $\mathcal{C}(P)$. If $P$ is a Heegaard surface, then $d(P)$ is the distance defined by Hempel [7]. We say $P$ is strongly irreducible or following the definition in [14], say $P$ is weakly incompressible if $d(P) \geq 2$, ie every compressing disk in $U$ intersects every compressing disk in $V$.

Let $Q$ be another closed orientable surface embedded in $M$. Let $g(Q)$ be the genus of $Q$. A theorem of Hartshorn [5] says that if $Q$ is incompressible and $P$ is a strongly 
irreducible Heegaard surface, then $d(P) \leq 2 g(Q)$. In [14], Scharlemann and Tomova showed that if both $P$ and $Q$ are connected, separating, bicompressible and strongly irreducible, then either $d(P) \leq 2 g(Q)$ or $P$ and $Q$ are well-separated or $P$ and $Q$ are isotopic. In particular, if both $P$ and $Q$ are strongly irreducible Heegaard surfaces, either $P$ and $Q$ are isotopic or $d(P) \leq 2 g(Q)$.

Combining Hartshorn's theorem and the theorem of Scharlemann and Tomova, we have the following Theorem.

Theorem 1.1 Suppose $M$ is a compact orientable irreducible 3-manifold and $P$ is a separating bicompressible and strongly irreducible (or weakly incompressible) surface in $M$. Let $Q$ be an embedded closed orientable surface in $M$ and suppose $Q$ is either incompressible or separating, bicompressible but strongly irreducible. Then either

(1) $d(P) \leq 2 g(Q)$, or

(2) after isotopy, $P_{t} \cap Q=\varnothing$ for all $t$, where $P_{t}(t \in[0,1])$ is a level surface in a sweep-out for $P$, see Section 2 for definition, or

(3) $P$ and $Q$ are isotopic.

Remark The statement of Theorem 1.1 is basically the same as the main theorem of [14]. If $Q$ is separating, bicompressible but strongly irreducible and $P_{t} \cap Q=\varnothing$ for all $t \in[0,1]$, then it is easy to see that $P$ and $Q$ are well-separated. Note that part (3) of the theorem never happens if $Q$ is incompressible.

In this paper, we give another proof of Theorem 1.1. Some arguments were originally used in a different proof of the main theorem by the author [9]. The motivation for this paper is a conjecture in [9] which generalizes both the main theorem of [9] and the theorem of Scharlemann and Tomova. We hope this proof and the techniques in [9; 10] can lead to a solution of this conjecture. Some arguments in the proof are similar to those in $[1 ; 14]$.

I would like to thank Marty Scharlemann for pointing out a mistake in an earlier version of the paper. The research was partially supported by NSF grant DMS-0406038.

\section{Saddle tangencies}

Notation Throughout this paper, we denote the interior of $X$ by $\operatorname{int}(X)$ for any space $X$. 
Let $P$ be a bicompressible surface and let $U$ and $V$ be the closure of the two components of $M-P$ as above. Let $P^{U}$ and $P^{V}$ be the possibly disconnected surfaces obtained by maximally compressing $P$ in $U$ and $V$ respectively. Since $M$ is irreducible, after capping off 2-sphere components by 3-balls, we may assume $P^{U}$ and $P^{V}$ do not contain 2-sphere components. Moreover, we may also assume $P^{U} \subset \operatorname{int}(U)$ and $P^{V} \subset \operatorname{int}(V)$. Since $P$ is strongly irreducible, as in Casson-Gordon [3], $P^{U}$ and $P^{V}$ are incompressible in $M$. Furthermore, $P^{U} \cup P^{V}$ bounds a submanifold $M_{P}$ of $M$ and $P$ is a strongly irreducible Heegaard surface of $M_{P}$. Note that if $U$ is a handlebody, then $P^{U}=\varnothing$. If $P$ is a Heegaard surface of $M$, then we may view $M_{P}=M$.

The surface $P$ cuts $M_{P}$ into a pair of compression bodies $U \cap M_{P}$ and $V \cap M_{P}$. There are a pair of properly embedded graphs $G^{U} \subset U \cap M_{P}$ and $G^{V} \subset V \cap M_{P}$ which are the spines of the two compression bodies. The endpoints of the graphs $G^{U}$ and $G^{V}$ lie in $P^{U}$ and $P^{V}$ respectively. Let $\Sigma_{U}=P^{U} \cup G^{U}$ and $\Sigma_{V}=P^{V} \cup G^{V}$, then $M_{P}-\left(\Sigma_{U} \cup \Sigma_{V}\right)$ is homeomorphic to $P \times(0,1)$. Throughout this paper, $\Sigma_{U}$ and $\Sigma_{V}$ are fixed.

We consider a sweepout $H: P \times(I, \partial I) \rightarrow\left(M_{P}, \Sigma_{U} \cup \Sigma_{V}\right)$, see [11], where $I=[0,1]$ and $\left.H\right|_{P \times(0,1)}$ is an embedding. We denote $H(P \times\{x\})$ by $P_{x}$ for any $x \in I$. We may assume $P_{0}=\Sigma_{U}, P_{1}=\Sigma_{V}$ and each $P_{x}(i \neq 0,1)$ is isotopic to $P$. To simplify notation, we will not distinguish $H(P \times(0,1))$ from $P \times(0,1)$.

Let $\pi: P \times I \rightarrow P$ be the projection. To simplify notation, we do not distinguish between an essential simple closed curve $\gamma$ in $P_{x}$ and the vertex represented by $\pi(\gamma)$ in the curve complex $\mathcal{C}(P)$.

Definition 2.1 Let $Q$ be a properly embedded compact surface in $M$. We say $Q$ is in regular position with respect to $P \times I$ if

(1) $Q \cap G^{U}$ and $Q \cap G^{V}$ consist of finitely many points and $Q$ is transverse to $P^{U} \cup P^{V}$ and

(2) $Q$ is transverse to each $P_{x}, x \in(0,1)$, except for finitely many critical levels $t_{1}, \ldots, t_{n} \in(0,1)$ and

(3) at each critical level $t_{i}, Q$ is transverse to $P_{t_{i}}$ except for a single saddle or center tangency.

If $x \in(0,1)$ is not one of the $t_{i}$ 's, then we say $x$ or $P_{x}$ is a regular level. Clearly every embedded surface $Q$ can be isotoped into a regular position.

Definition 2.2 We say $Q$ is irreducible with respect to $P \times I$ if 


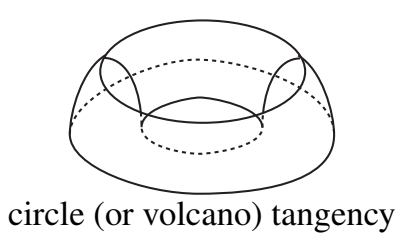

(a)

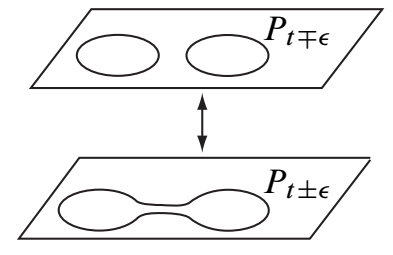

(b)

Figure 2.1

(1) $Q$ is in regular position with respect to $P \times I$ and

(2) at each regular level $P_{x}$, if a component $\gamma$ of $Q \cap P_{x}$ is trivial in $P_{x}$, then $\gamma$ is also trivial in $Q$.

In this section, we assume $Q$ is irreducible with respect to the sweepout $P \times I$. We first perform some isotopy on $Q$ to eliminate center tangencies and trivial intersection curves. Lemma 2.1 can be viewed as a special case of a theorem of Thurston [15] and [4, Theorem 7.1].

Lemma 2.1 Let $Q$ be an embedded surface in $M$ and suppose $Q$ is irreducible with respect to the sweepout $P \times I$. Then, one can perform an isotopy on $Q$ so that

(1) $Q \cap\left(G^{U} \cup G^{V}\right)$ consists of finitely many points, $Q$ is transverse to $P^{U} \cup P^{V}$, and $Q \cap\left(P^{U} \cup P^{V}\right)$ consists of curves essential in $Q$;

(2) $Q$ is transverse to each $P_{x}, x \in(0,1)$, except for finitely many critical levels $t_{1}, \ldots, t_{n} \in(0,1)$;

(3) at each critical level $t_{i}, Q$ is transverse to $P_{t_{i}}$ except for a saddle or circle tangency, as shown in Figure 2.1(a);

(4) at each regular level $x$, every component of $Q \cap P_{x}$ is an essential curve in $P_{x}$.

Proof Since $P^{U} \cup P^{V}$ is incompressible in $M$ and $M$ is irreducible, after some standard isotopy we may assume condition (1) in the lemma holds.

Note that the intersection of $Q$ with $P \times I$ yields a (singular) foliation of $Q \cap M_{P}$ with each leaf a component of $Q \cap P_{x}$ for some $x \in I$. A singular point in the foliation is either a point in $Q \cap\left(G^{U} \cup G^{V}\right)$ or a saddle or center tangency.

Let $x$ be a regular level and suppose a component $\gamma$ of $Q \cap P_{x}$ is trivial in $P_{x}$. Suppose $\gamma$ is innermost in $P_{x}$, ie the disk bounded by $\gamma$ in $P_{x}$ does not contain 
any other intersection curve with $Q$. Since $Q$ is irreducible with respect to $P \times I$, $\gamma$ bounds a disk $D_{\gamma}$ in $Q$. If the induced foliation on $D_{\gamma}$ contains more than one singular point, since $\gamma$ is trivial in $P_{x}$, we can construct a disk $D^{\prime} \subset P \times(x-\epsilon, x+\epsilon)$ for some small $\epsilon$ such that

(1) $\partial D^{\prime}=\gamma$,

(2) the induced foliation of $D^{\prime} \cap(P \times I)$ consists of parallel circles except for a singular point corresponding to a center tangency,

(3) $\left(Q-D_{\gamma}\right) \cup D^{\prime}$ is embedded in $M$ and irreducible with respect to $P \times I$.

Since $M$ is irreducible, $\left(Q-D_{\gamma}\right) \cup D^{\prime}$ is isotopic to $Q$. Moreover, the induced foliation on $\left(Q-D_{\gamma}\right) \cup D^{\prime}$ has fewer singular points. So after finitely many such operations, we may assume that for any regular level $x$ and for any component $\gamma$ of $Q \cap P_{x}$ that is trivial in $P_{x}$, the disk bounded by $\gamma$ in $Q$ lies in $M_{P}$ and is transverse to $P \times(0,1)$ except for a single center tangency.

Let $t$ be a critical level and suppose $Q \cap P_{t}$ contains a saddle tangency. Let $\epsilon$ be a sufficiently small number. So the component of $Q \cap(P \times[t-\epsilon, t+\epsilon])$ that contains the saddle tangent point is a pair of pants $F$. Figure 2.1(b) is a picture of the curves changing from $F \cap P_{t-\epsilon}$ to $F \cap P_{t+\epsilon}$.

We first claim that at most one component of $\partial F$ is trivial in the corresponding level surface $P_{t \pm \epsilon}$. Let $\gamma_{1}, \gamma_{2}$ and $\gamma_{3}$ be the 3 components of $\partial F$ and suppose $\gamma_{1}$ and $\gamma_{2}$ are both trivial in the corresponding level surfaces. Then by the change of $F \cap P_{x}$ near the saddle tangency as shown in Figure 2.1(b), $\gamma_{3}$ must also be trivial in the corresponding level surface $P_{t \pm \epsilon}$. Since $Q$ is irreducible with respect to $P \times I, \gamma_{1}$ and $\gamma_{2}$ bound disks $D_{1}$ and $D_{2}$ in $Q$ respectively. By the assumption above, the disk $D_{i}$ does not contain any saddle tangency and hence $F \cap D_{i}=\gamma_{i}, i=1,2$. Thus $F \cup D_{1} \cup D_{2}$ is a disk in $Q$ bounded by $\gamma_{3}$ and $F \cup D_{1} \cup D_{2}$ contains a saddle tangent point. This contradicts the assumption above. Thus at most one component of $\partial F$ is trivial in $P_{t \pm \epsilon}$.

Let $F$ and $\gamma_{i}$ be as above. Suppose $\gamma_{1}$ and $\gamma_{2}$ lie in $P_{t-\epsilon}$ and $\gamma_{3}$ lies in $P_{t+\epsilon}$. If $\gamma_{1}$ is trivial in $P_{t-\epsilon}$ and let $D_{1}$ be the disk in $Q$ bounded by $\gamma_{1}$, then $F \cap D_{1}=\gamma_{1}$ as above and $F \cup D_{1}$ is an annulus in $Q$ bounded by $\gamma_{2} \cup \gamma_{3}$. Since $D_{1}$ is isotopic to a disk in $P_{t-\epsilon}$, we can first push $D_{1}$ into $P \times[t-\epsilon, t+\epsilon]$, then as shown in Figure 2.2(a), we may perform another isotopy on $Q$ canceling the center tangency in $D_{1}$ and the saddle tangency in $F$. If $\gamma_{3}$ is trivial in $P_{t+\epsilon}$, by the assumption above, both $\gamma_{1}$ and $\gamma_{2}$ are essential in $P_{t-\epsilon}$. Hence $\gamma_{1}$ and $\gamma_{2}$ must be parallel in $P_{t-\epsilon}$. Let $D_{3}$ be the disk in $Q$ bounded by $\gamma_{3}$. As above, $F \cap D_{3}=\gamma_{3}$ and $F \cup D_{3}$ is an annulus in $Q$ bounded by $\gamma_{1} \cup \gamma_{2}$. Since $D_{3}$ is isotopic to the disk in $P_{t+\epsilon}$ bounded by $\gamma_{3}$, 


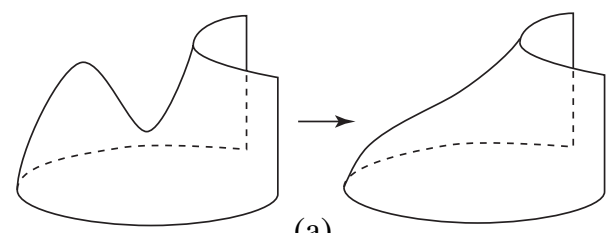

(a)

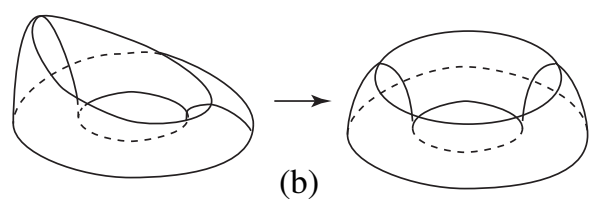

Figure 2.2

we can first push the annulus $F \cup D_{3}$ into a $\partial$-parallel annulus in $P \times[t-\epsilon, t+\epsilon]$. Then an isotopy as shown in Figure 2.2(b) can cancel the center tangency in $D_{3}$ and the saddle tangency in $F$, changing $F \cup D_{3}$ into an annulus with a circle (or volcano) tangency. Note that the circle tangency is an essential curve in the corresponding level surface $P_{x}$.

Note that condition (1) of the lemma implies that for a small $\epsilon, Q \cap P_{\epsilon}$ and $Q \cap P_{1-\epsilon}$ consist of essential curves in $P_{\epsilon}$ and $P_{1-\epsilon}$ respectively. Since $Q$ is not a $2-$ sphere, a curve of $Q \cap P_{x}$ that is trivial in $P_{x}$ will eventually meet and cancel with a saddle tangency. Thus after a finite number of isotopies as above, we can eliminate all the curves of $Q \cap P_{x}$ that are trivial in $P_{x}$, and get a surface $Q$ satisfying all the conditions in the lemma.

Note that a circle tangency does not create any singularity in the foliation of $Q \cap M_{P}$ induced from $P \times I$. Thus, if $Q$ satisfies the conditions in Lemma 2.1, a singular point in the foliation of $Q \cap M_{P}$ corresponds to either a saddle tangency or a point in $Q \cap\left(G^{U} \cup G^{V}\right)$. It is possible that $Q$ does not intersect $M_{P}=P \times I$, ie $P_{t} \times Q=\varnothing$ for all $t$, after isotopy.

Lemma 2.2 Let $P$ and $Q$ be as above and assume $Q$ satisfies the conditions in Lemma 2.1. Suppose $Q \cap \Sigma_{U} \neq \varnothing$ and $Q \cap \Sigma_{V} \neq \varnothing$. Then the distance $d(P)=$ $d(\mathcal{U}, \mathcal{V}) \leq 2 g(Q)$.

Proof Since $Q$ is connected and $P$ is separating, $Q \cap \Sigma_{U} \neq \varnothing$ and $Q \cap \Sigma_{V} \neq \varnothing$ imply $Q \cap P_{t} \neq \varnothing$ for every $t$. 
Claim 1 Let $t$ be a critical level and $\epsilon$ a sufficiently small number. Let $\sigma$ and $w$ be any components of $Q \cap P_{t-\epsilon}$ and $Q \cap P_{t+\epsilon}$ respectively. Then $d(\sigma, w) \leq 1$.

Proof of Claim 1 The claim is obvious if $P_{t}$ contains a circle tangency. So we suppose $P_{t}$ contains a saddle tangency. Let $F$ be the component of $Q \cap(P \times[t-\epsilon, t+\epsilon])$ that contains the saddle tangency. Then $F$ is a pair of pants and all other components of $Q \cap(P \times[t-\epsilon, t+\epsilon])$ are essential vertical annuli in $P \times[t-\epsilon, t+\epsilon]$. If $\sigma$ is a boundary curve of a vertical annulus, then $\sigma$ is isotopic to a component of $Q \cap P_{t+\epsilon}$ and hence $d(\sigma, w) \leq 1$ for any curve $w$ in $Q \cap P_{t+\epsilon}$. If neither $\sigma$ nor $w$ is a boundary curve of a vertical annulus, then $\sigma$ and $w$ are components of $\partial F$ and $d(\sigma, w)=1$ as shown in Figure 2.1(b).

Let $s_{0}<\cdots<s_{n}$ be a collection of regular levels such that $s_{0}=\delta, s_{n}=1-\delta$ for a small $\delta$ and there is exactly one saddle or circle tangency in each $P \times\left(s_{i}, s_{i+1}\right)$. Let $\Gamma_{i}=Q \cap P_{s_{i}}$ for each $i$.

Recall that $P_{0}=\Sigma_{U}=P^{U} \cup G^{U}$ and $P_{1}=\Sigma_{V}=P^{V} \cup G^{V}$. Since $s_{0}=\delta$ for a small $\delta$, we may assume $d\left(\mathcal{U}, \Gamma_{0}\right)$ is either 0 or 1 , and if $d\left(\mathcal{U}, \Gamma_{0}\right)=1$ then $d(\mathcal{U}, \sigma)=1$ for any component $\sigma$ of $\Gamma_{0}$. Similarly, $d\left(\mathcal{V}, \Gamma_{n}\right)$ is either 0 or 1 , and if $d\left(\mathcal{V}, \Gamma_{n}\right)=1$ then $d(\mathcal{V}, w)=1$ for any component $w$ of $\Gamma_{n}$.

Suppose $d(\mathcal{U}, \mathcal{V})>2 g(Q)$ and hence $d(\mathcal{U}, \mathcal{V})>2$. Let $k$ be the smallest integer such that $d\left(\mathcal{U}, \Gamma_{k}\right) \neq 0$ and $l$ the largest integer such that $d\left(\Gamma_{l}, \mathcal{V}\right) \neq 0$. Since $d\left(\mathcal{U}, \Gamma_{0}\right) \leq 1$ and $d\left(\mathcal{V}, \Gamma_{n}\right) \leq 1$, by Claim 1 above, $d\left(\mathcal{U}, \Gamma_{k}\right)=d\left(\Gamma_{l}, \mathcal{V}\right)=1$ and $k \leq l$. Without loss of generality, we assume $k<l$. Next we show that every curve in $\Gamma_{k}$ is essential in $Q$. Suppose a curve $\gamma$ in $\Gamma_{k}$ is trivial in $Q$ and let $D$ be the disk bounded by $\gamma$ in $Q$. Since $P^{U}$ and $P^{V}$ are incompressible, we may assume $D \subset M_{P}$. Since $P$ is a strongly irreducible Heegaard surface of $M_{P}$, by the no-nesting lemma of Scharlemann [12, Lemma 2.2], $\gamma$ must bound a disk in one of the two compression bodies, ie either $\gamma \in \mathcal{U}$ or $\gamma \in \mathcal{V}$. However, $\gamma \in \mathcal{U}$ contradicts $d\left(\mathcal{U}, \Gamma_{k}\right) \neq 0$, and $\gamma \in \mathcal{V}$ contradicts $d(\mathcal{U}, \mathcal{V})>2$. Thus every curve in $\Gamma_{k}$ must be essential in $Q$. Similarly every curve in $\Gamma_{l}$ is also essential in $Q$.

Let $Q^{\prime}=Q \cap\left(P \times\left[s_{k}, s_{l}\right]\right)$, and let $U^{\prime}$ and $V^{\prime}$ be the two components of $M-$ $P \times\left(s_{k}, s_{l}\right)$ containing $G^{U}$ and $G^{V}$ respectively, $F_{U}=Q \cap U^{\prime}$ and $F_{V}=Q \cap V^{\prime}$. Since $\Gamma_{k}$ and $\Gamma_{l}$ are essential in $Q, F_{U}, Q^{\prime}$ and $F_{V}$ are essential subsurfaces of $Q=F_{U} \cup Q^{\prime} \cup F_{V}$.

Claim 2 Let $\sigma_{k}$ be any component of $\Gamma_{k}$, then $d\left(\sigma_{k}, \mathcal{U}\right) \leq 1$. 
Proof of Claim 2 By the definition of $k$ and the argument above, Claim 2 holds if $k=0$. If $k>0$, then $d\left(\mathcal{U}, \Gamma_{k-1}\right)=0$ and $d\left(\mathcal{U}, \Gamma_{k}\right)=1$. Let $w$ be a component of $\Gamma_{k-1}$ that represents a vertex in $\mathcal{U}$. By Claim 1, for any component $\sigma_{k}$ of $\Gamma_{k}$, $d\left(\sigma_{k}, \mathcal{U}\right) \leq d\left(\sigma_{k}, w\right) \leq 1$.

Claim 3 There is a component $\sigma_{k}$ of $\Gamma_{k}$ and a component $\sigma_{l}$ of $\Gamma_{l}$ such that $d\left(\sigma_{k}, \sigma_{l}\right) \leq-\chi\left(Q^{\prime}\right)$.

Proof of Claim 3 Let $t_{1}<\cdots<t_{N}$ be the levels in $\left(s_{k}, s_{l}\right)$ that contain the saddle tangencies. For a sufficiently small $\epsilon, P \times\left[t_{i}+\epsilon, t_{i+1}-\epsilon\right]$ contains no saddle tangency for each $i$ (to simplify notation we set $t_{0}+\epsilon=s_{k}$ and $t_{N+1}-\epsilon=s_{l}$ ). So by the conditions in Lemma 2.1, $Q \cap\left(P \times\left[t_{i}+\epsilon, t_{i+1}-\epsilon\right]\right)$ consists of annuli for each $i=0, \ldots, N$. If $Q \cap\left(P \times\left[t_{i}+\epsilon, t_{i+1}-\epsilon\right]\right)$ consists of $\partial$-parallel annuli, then $Q \cap P_{t}=\varnothing$ for some $t$ after isotopy, a contradiction to our assumption at the beginning. Thus an annulus component $A_{i}$ of $Q \cap\left(P \times\left[t_{i}+\epsilon, t_{i+1}-\epsilon\right]\right)$ is vertical. We choose $\gamma_{i}$ to be a meridian circle in $A_{i}$ for each $i$ and assume $\sigma_{k}=\gamma_{0}=A_{0} \cap P_{s_{k}} \subset \Gamma_{k}$ and $\sigma_{l}=\gamma_{N}=A_{N} \cap P_{s_{l}} \subset \Gamma_{l}$. Since each $A_{i}$ is vertical, $\gamma_{i}$ is parallel to a component of $Q \cap P_{t_{i+1}-\epsilon}$. Similarly $\gamma_{i+1}$ is parallel to a component of $Q \cap P_{t_{i+1}+\epsilon}$. By Claim $1, d\left(\gamma_{i}, \gamma_{i+1}\right) \leq 1$ and hence $d\left(\sigma_{k}, \sigma_{l}\right)=d\left(\gamma_{0}, \gamma_{N}\right) \leq N$. Moreover, since the only singular points in the induced foliation of $Q^{\prime}$ are the saddle tangencies, by a standard index argument, $-\chi\left(Q^{\prime}\right)=N$ and hence $d\left(\sigma_{k}, \sigma_{l}\right) \leq-\chi\left(Q^{\prime}\right)$.

Since $Q^{\prime}, F_{U}$ and $F_{V}$ are essential subsurfaces of $Q, \chi\left(Q^{\prime}\right) \geq \chi(Q)$. By Claim 2, $d\left(\sigma_{k}, \mathcal{U}\right) \leq 1$ and similarly $d\left(\sigma_{l}, \mathcal{V}\right) \leq 1$. Therefore, $d(\mathcal{U}, \mathcal{V}) \leq d\left(\mathcal{U}, \sigma_{k}\right)+d\left(\sigma_{k}, \sigma_{l}\right)+$ $d\left(\sigma_{l}, \mathcal{V}\right) \leq 1-\chi\left(Q^{\prime}\right)+1 \leq 2-\chi(Q)=2 g(Q)$.

Lemma 2.2 implies that if $d(\mathcal{U}, \mathcal{V})$ is large, then not every $Q$ can be put into a position satisfying all the hypotheses of Lemma 2.2.

Corollary 2.1 Let $P$ and $Q$ be as in Theorem 1.1. Then Theorem 1.1 holds if $Q$ is incompressible.

Proof If $Q$ is incompressible, then $Q$ can be isotoped to be irreducible with respect to $P \times I$. Moreover, if $Q \cap \Sigma_{U}=\varnothing$, then since $Q$ is incompressible, $Q$ can be isotoped out of the compression body $M_{P}-N\left(\Sigma_{U}\right)$. Hence $Q \cap M_{P}=\varnothing$ after isotopy and part (2) of Theorem 1.1 holds. Now Corollary 2.1 follows from Lemma 2.1 and Lemma 2.2 . 


\section{The graphics of sweepouts}

In this section, we suppose $Q$ is separating, bicompressible and strongly irreducible.

Let $X$ and $Y$ be the closure of the 2 components of $M-Q$. Let $Q^{X}$ and $Q^{Y}$ be the possibly disconnected surfaces obtained by maximal compressing $Q$ in $X$ and $Y$ respectively and capping off 2 -sphere components by 3 -balls. Similar to the argument on $P^{U}$ and $P^{V}$ above, we may assume $Q^{X} \subset \operatorname{int}(X)$ and $Q^{Y} \subset \operatorname{int}(Y)$ are incompressible in $M$. Furthermore, $Q^{X} \cup Q^{Y}$ bounds a submanifold $M_{Q}$ of $M$ and $Q$ is a strongly irreducible Heegaard surface of $M_{Q}$. If $X$ is a handlebody, then $Q^{X}=\varnothing$. If $Q$ is a Heegaard surface of $M$, we may view $M_{Q}=M$.

As in Section 2, the surface $Q$ cuts $M_{Q}$ into a pair of compression bodies $X \cap M_{Q}$ and $Y \cap M_{Q}$. Let graphs $G^{X} \subset X \cap M_{Q}$ and $G^{Y} \subset Y \cap M_{Q}$ be the spines of the two compression bodies and let $\Sigma_{X}=Q^{X} \cup G^{X}$ and $\Sigma_{Y}=Q^{Y} \cup G^{Y}$. Then $M_{Q}-\left(\Sigma_{X} \cup \Sigma_{Y}\right)$ is homeomorphic to $Q \times(0,1)$.

Now we consider the two sweepouts $H: P \times(I, \partial I) \rightarrow\left(M_{P}, \Sigma_{U} \cup \Sigma_{V}\right)$ and $H^{\prime}: Q \times$ $(I, \partial I) \rightarrow\left(M_{Q}, \Sigma_{X} \cup \Sigma_{Y}\right)$. Let $P_{t}=H(P \times\{t\})$ and $Q_{t}=H^{\prime}(Q \times\{t\}), t \in I$. We may assume $Q_{0}=\Sigma_{X}, Q_{1}=\Sigma_{Y}$ and $Q_{x}$ is isotopic to $Q$ for each $x \in(0,1)$.

The graphic $\Lambda$ of the sweepouts, defined in [11], is the set of points $(s, t) \in(0,1) \times(0,1)$ such that $P_{s}$ is not transverse to $Q_{t}$. We briefly describe the graphic below and refer to [11] for more details. As in [11], Cerf theory implies that after some isotopy, we may assume that $\Lambda$ is a graph in $(0,1) \times(0,1)$ whose edges are the set of points $(s, t)$ for which $P_{s}$ is transverse to $Q_{t}$ except for a single saddle or center tangency. There are two types of vertices in $\Lambda$, birth-and-death vertices and crossing vertices, as shown in Figure 3.1(a). Moreover, each arc $(0,1) \times\{x\}$ contains at most one vertex, $x \in(0,1)$. The complement of $\Lambda,(0,1) \times(0,1)-\Lambda$, is a finite collection of regions. Note that for every $(s, t)$ in $(0,1) \times(0,1)-\Lambda, P_{s}$ is transverse to $Q_{t}$, and for any two points $(s, t)$ and $\left(s^{\prime}, t^{\prime}\right)$ in the same region, $P_{s} \cap Q_{t}$ and $P_{s^{\prime}} \cap Q_{t^{\prime}}$ have the same intersection pattern.

Let $(s, t) \in(0,1) \times(0,1)-\Lambda$. Suppose there are disks or annuli $D_{P} \subset P_{s}$ and $D_{Q} \subset Q_{t}$ with $D_{P} \cap D_{Q}=\partial D_{P}=\partial D_{Q} \subset P_{S} \cap Q_{t}$. Suppose $D_{P}$ is parallel to $D_{Q}$ (fixing $\partial D_{P}=\partial D_{Q}$ ) in $M$ and suppose $D_{P} \cup D_{Q}$ bounds a 3-ball or solid torus $E$. Moreover, suppose $Q_{t} \cap E=D_{Q}$. Then we can perform an isotopy on $Q_{t}$ by pushing $D_{Q}$ across $E$ and remove the intersection $\partial D_{P}=\partial D_{Q}$. This isotopy is the same as the operation that changes $Q_{t}$ to $\left(Q_{t}-D_{Q}\right) \cup D_{P}$ and then perturbs the resulting surface. We call such an isotopy a trivial isotopy on $Q_{t}$ at $P_{s}$. We may view a trivial isotopy on $Q_{t}$ as associated with the disk or annulus $D_{Q} \subset Q_{t}$. Suppose we are to perform another trivial isotopy on $Q_{t}$ at $P_{S^{\prime}}$ and let $D_{Q}^{\prime} \subset Q_{t}$ be the disk or annulus 


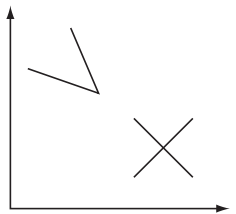

(a)

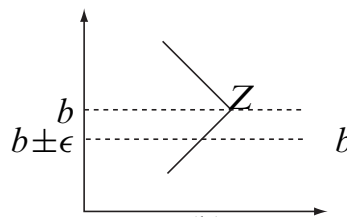

(b)

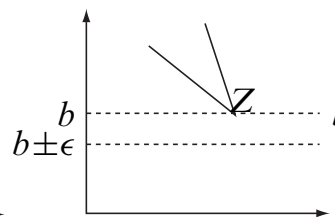

(c)

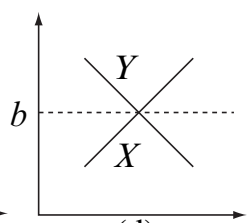

(d)

Figure 3.1

in the isotopy as above. Then $D_{Q}$ and $D_{Q}^{\prime}$ are either disjoint or nested in $Q_{t}$. Thus either the two trivial isotopies are disjoint or we can view one isotopy as a middle step of the other.

Labelling For any $Q_{t}$, we use $X_{t}$ (resp. $Y_{t}$ ) to denote the component of $M-Q_{t}$ that contains $\Sigma_{X}$ (resp. $\left.\Sigma_{Y}\right)$. We label a region, ie a component of $(0,1) \times(0,1)-\Lambda$, $X$ (resp. $Y$ ) if for a point $(s, t)$ in the region, either (1) there is a component of $P_{s} \cap Q_{t}$ that is trivial in $P_{s}$ but bounds an essential disk in $X_{t}$ (resp. $Y_{t}$ ), or (2) $\Sigma_{U}$ or $\Sigma_{V}$ lies in $Y_{t}$ (resp. $X_{t}$ ) after some trivial isotopies on $Q_{t}$ at finitely many regular levels $P_{X}$. We label $t \in(0,1) X$ (resp. $Y$ ) if the horizontal line segment $(0,1) \times\{t\}$ intersects a region labelled $X$ (resp. $Y$ ). Note that since a trivial isotopy does not increase $\left|\Sigma_{U} \cap Q_{t}\right|$ or $\left|\Sigma_{V} \cap Q_{t}\right|$, if $t$ is not labelled, $Q_{t} \cap \Sigma_{U} \neq \varnothing$ and $Q_{t} \cap \Sigma_{V} \neq \varnothing$ after any trivial isotopies.

Lemma 3.1 Either Theorem 1.1 holds or for a sufficiently small $\delta>0, \delta$ is labelled $X$ and $1-\delta$ is labelled $Y$.

Proof For a sufficiently small $\delta>0, H^{\prime}(Q \times[0, \delta])$ is a small neighborhood of $\Sigma_{X}=Q^{X} \cup G^{X}$. If $P_{s} \cap G^{X} \neq \varnothing$ for some $s$, then by definition, $\delta$ is labelled $X$ for a sufficiently small $\delta$. Suppose $\delta$ is not labelled $X$, then the graph $G^{X}$ must be disjoint from $M_{P}=H(P \times I)$. Moreover, if $Q^{X} \cap P_{t}=\varnothing$ for some $t$ after isotopy, since $Q^{X}$ is incompressible, we can isotope $Q^{X}$ out of the two compression bodies $M_{P}-P_{t}$. Hence, $Q_{\delta} \cap M_{P}=\varnothing$ after isotopy and part (2) of Theorem 1.1 holds. If $Q^{X} \cap P_{t} \neq \varnothing$ for all $t$, since $Q^{X}$ is incompressible, by Corollary 2.1, $d(P) \leq 2 g\left(Q^{X}\right) \leq 2 g(Q)$ and Theorem 1.1 follows. The proof for $1-\delta$ is similar.

Lemma 3.2 Either Theorem 1.1 holds or no $t \in(0,1)$ is labelled both $X$ and $Y$.

Proof We first remark that if $\Sigma_{U} \subset Y_{t}$ then one cannot move $\Sigma_{U}$ to $X_{t}$ by a trivial isotopy, since if this happens, then $\Sigma_{U}$ must lie in $E$, where $E$ is the 3-ball or solid 
torus in the trivial isotopy described above. However, since $g(P) \geq 2$ and $P$ is strongly irreducible, $\Sigma_{U}$ cannot lie in a 3-ball or solid torus by [3]. So by our labelling, if $t$ is labelled both $X$ and $Y$, then one can always find $s_{1} \neq s_{2}$ such that $P_{s_{1}}$ and $P_{s_{2}}$ are transverse to $Q_{t}$ and one of the following three cases occurs.

Case 1 A component of $P_{S_{1}} \cap Q_{t}$ contains a curve bounding an essential disk $D_{X}$ in $X_{t}$ and a component of $P_{s_{2}} \cap Q_{t}$ contains a curve bounding an essential disk $D_{Y}$ in $Y_{t}$. In this case, since $s_{1} \neq s_{2}, \partial D_{X} \cap \partial D_{Y}=\varnothing$ in $Q_{t}$, which contradicts the assumption that $Q$ is strongly irreducible.

Case 2 After trivial isotopies, $\Sigma_{U} \subset Y_{t}$ and $\Sigma_{V} \subset X_{t}$. This means that $Q_{t} \subset$ $P \times(0,1) \subset M_{P}$ and $Q_{t}$ separates $\Sigma_{U}$ and $\Sigma_{V}$ in $M_{P}$. The proof for this case is similar to that of [14, Lemma 2.3]. If $Q_{t}$ is incompressible in $P \times(0,1)$, then $Q_{t}$ is isotopic to $P$ and Theorem 1.1 holds. If $Q_{t}$ is compressible on both sides in $P \times(0,1)$, similar to the construction of $M_{Q}$ earlier, by maximally compressing $Q_{t}$ in $P \times(0,1)$ on both sides and capping off $2-$ sphere components, we obtain a submanifold $M_{Q}^{\prime}$ of $P \times(0,1)$ such that $Q_{t}$ is a strongly irreducible Heegaard surface of $M_{Q}^{\prime}$. Moreover, by [3], $\partial M_{Q}^{\prime}$ is incompressible in $P \times(0,1)$. So each component of $\partial M_{Q}^{\prime}$ is parallel to $P$ and $M_{Q}^{\prime}$ must be a product of $P$ and an interval. Thus we can view $Q_{t}$ as a strongly irreducible Heegaard surface of a product $P \times[0,1]$. By Scharlemann-Thompson [13], either $Q_{t}$ is isotopic to $P$ or $Q_{t}$ cuts $P \times[0,1]$ into a handlebody and a compression body. In the later case, both $\Sigma_{U}$ and $\Sigma_{V}$ lie in $Y_{t}$ (or both in $X_{t}$ ), a contradiction. If $Q_{t}$ is compressible on only one side, say the $X_{t}$ side. Then after maximally compressing $Q_{t}$ in $P \times(0,1)$ on the $X_{t}$ side, one obtains an incompressible surface $Q^{\prime}$ in $P \times(0,1)$ (note that $Q^{\prime} \neq \varnothing$ as $\Sigma_{V} \subset X_{t}$ ). Thus $Q^{\prime}$ is incompressible in $P \times(0,1)$ and must be parallel to $P$. Moreover, since $Q_{t}$ is connected and separating, $Q^{\prime}$ is a single parallel copy of $P$. So $Q_{t}$ and $Q^{\prime}$ bound a compression body $W$ in $P \times(0,1)$, and $Q_{t}$ is bicompressible in the submanifold $Y_{t} \cup W$ of $M$. Since $Q_{t}$ is strongly irreducible, Casson-Gordon [3] implies that $Q^{\prime}$ is incompressible in $Y_{t} \cup W$. However, since $Q^{\prime}$ is parallel to $P$, this contradicts the assumption that $P$ is compressible on both sides.

Case 3 After trivial isotopies, $\Sigma_{U} \subset Y_{t}$ and a component of $P_{s_{1}} \cap Q_{t}$ contains a curve $\gamma$ that is trivial in $P_{s_{1}}$ and bounds an essential disk $D$ in $Y_{t}$. Note that if a component of $P_{s_{1}} \cap Q_{t}$ also bounds an essential disk in $X_{t}$, then this contradicts that $Q$ is strongly irreducible as in case (1). Thus, after some isotopy on $Q_{t}$, we may assume that $\gamma$ is innermost in $P_{S_{1}}$ and the disk $D$ bounded by $\gamma$ in $P_{s_{1}}$ is an essential disk in $Y_{t}$. Since $\Sigma_{U} \subset Y_{t}$ and $D \subset Y_{t}-\Sigma_{U}$, by maximally compressing $Q_{t}$ in $Y_{t}-\Sigma_{U}$ and capping off 2-sphere components, we obtained a (possibly disconnected) surface $Q_{t}^{Y}$. Note that $Q_{t}^{Y} \neq \varnothing$ because $\Sigma_{U}$ is not contained in a 3-ball. Since $Q_{t}$ 
is strongly irreducible, by [3], $Q_{t}^{Y}$ is incompressible in $M-\Sigma_{U}$. Note that if $P$ is a Heegaard surface of a closed manifold $M$, this is already a contradiction since $Q_{t}^{Y}$ lies in the handlebody $M-N\left(\Sigma_{U}\right)$ and cannot be incompressible. $Q_{t}^{Y}$ cuts $Y_{t}$ into $H_{1}$ and $H_{2}$, where $H_{2}$ is the compression body bounded by $Q_{t}$ and $Q_{t}^{Y}$. Since the compressions on $Q_{t}$ are disjoint from $\Sigma_{U}$ and since $\Sigma_{U}$ does not lie in a 3-ball, $\Sigma_{U} \cap H_{2}=\varnothing$. Hence $\Sigma_{U} \subset H_{1}$. Since $Q_{t}^{Y}$ is incompressible in $M-\Sigma_{U}$, we can push $Q_{t}^{Y}$ out of the compression body $M_{P}-N\left(\Sigma_{U}\right)$ or equivalently push $M_{P}-N\left(\Sigma_{U}\right)$ into $H_{1}$. So we can isotope $M_{P}$ into $H_{1}$. In particular, $Q_{t} \cap M_{P}=\varnothing$ after isotopy and part (2) of Theorem 1.1 holds.

Lemma 3.3 If $t \in(0,1)$ has no label and $(0,1) \times\{t\}$ contains no vertex of $\Lambda$, then $Q_{t}$ is irreducible with respect to $P \times I$ and Theorem 1.1 holds.

Proof Since $(0,1) \times\{t\}$ contains no vertex of $\Lambda, Q_{t}$ is in regular position with respect to $P \times I$. For any $(s, t) \notin \Lambda$, suppose a curve $\gamma$ in $P_{s} \cap Q_{t}$ is trivial in $P_{s}$. If $\gamma$ is an essential curve in $Q_{t}$, by assuming $\gamma$ to be an innermost such curve, the disk bounded by $\gamma$ in $P_{s}$ can be isotoped to be an essential disk in either $X_{t}$ or $Y_{t}$. Since $t \in(0,1)$ has no label, $\gamma$ must be trivial in $Q_{t}$. Thus by definition, $Q_{t}$ is irreducible with respect to $P \times I$. So after isotopy we may assume $Q$ satisfies the conditions in Lemma 2.1. Moreover, since $t$ has no label, $Q_{t} \cap \Sigma_{U} \neq \varnothing$ and $Q_{t} \cap \Sigma_{V} \neq \varnothing$ after the isotopy in the proof of Lemma 2.1. So Theorem 1.1 follows from Lemma 2.2.

Suppose Theorem 1.1 is not true. Then by Lemma 3.1, for a small $\delta, \delta$ is labelled $X$ and $1-\delta$ is labelled $Y$. As $t$ changes from $\delta$ to $1-\delta$, the label changes from $X$ to $Y$. So by Lemma 3.2 and Lemma 3.3 , there must be a number $b \in(0,1)$ such that

(1) $(0,1) \times\{b\}$ contains a vertex of $\Lambda$ and

(2) $b$ has no label and

(3) $b-\epsilon$ is labelled $X$ and $b+\epsilon$ is labelled $Y$ for sufficiently small $\epsilon>0$.

Let $Z=(a, b)$ be the vertex of $\Lambda$ in $(0,1) \times\{b\}$. If $Z$ is a birth-and-death vertex, then since no region that intersects $(0,1) \times\{b\}$ is labelled, as shown in Figure 3.1(b) and (c), after perturbing $(0,1) \times\{b\}$ a little, we can find a line segment $(0,1) \times\{b \pm \epsilon\}$ that does not intersect any labelled region, a contradiction to our assumption above. Therefore, $Z=(a, b)$ must be a crossing vertex. Figure 3.1(d) is a picture of $Z$.

Since $Z=(a, b)$ is a crossing vertex, as explained in [11] (see Kobayashi-Saeki [8, Figure 2.6]), $P_{a}$ is transverse to $Q_{b}$ except for two saddle tangencies. Since $b$ is not labelled, for any $s \neq a$ in $(0,1)$, either (1) $P_{s} \cap Q_{b}$ contains a single center or saddle tangency or (2) $P_{s}$ is transverse to $Q_{b}$ and if a component of $P_{s} \cap Q_{b}$ is trivial 
in $P_{s}$ then it is also trivial in $Q_{b}$. Moreover, after trivial isotopies, $Q_{b} \cap \Sigma_{U} \neq \varnothing$ and $Q_{b} \cap \Sigma_{V} \neq \varnothing$. Since $P$ is separating and $Q$ is connected, this implies that $Q_{b} \cap P_{s} \neq \varnothing$ for all $s \in I$.

Now we consider $Q_{b} \cap(P \times[a-\epsilon, a+\epsilon])$ for a small $\epsilon$. Let $F$ be the union of the components of $Q_{b} \cap(P \times[a-\epsilon, a+\epsilon])$ that contain the two saddle tangencies. Thus $F$ is either the union of two disjoint pairs of pants or a connected surface with $\chi(F)=-2$. All other components of $Q_{b} \cap(P \times[a-\epsilon, a+\epsilon])$, denoted by $A_{1}, \ldots, A_{m}$, are vertical annuli in $P \times[a-\epsilon, a+\epsilon]$.

Next we consider the case that a component of $Q_{b} \cap P_{a \pm \epsilon}$ is trivial in $P_{a \pm \epsilon}$. If a component $\gamma$ of $\partial A_{i}, i=1, \ldots, m$, is trivial and innermost in $P_{a \pm \epsilon}$, then by our assumption, $\gamma$ bounds a disk $D_{\gamma}$ in $Q_{b}$. We can perform a trivial isotopy on $Q_{b}$ by pushing the disk $D_{\gamma} \cup A_{i}$ away from $P \times[a-\epsilon, a+\epsilon]$. Thus, after a finite number of such operations, we may assume the boundary of every annular component $A_{i}$ is essential in $P_{a \pm \epsilon}$.

Suppose a component $\gamma$ of $\partial F$ is an innermost trivial curve in $P_{a \pm \epsilon}$. So $\gamma$ bounds a disk $D_{\gamma}$ in $Q_{b}$. If $D_{\gamma}$ contains a component of $F$, then as in the proof of Lemma 2.1, after replacing $D_{\gamma}$ by a disk which is transverse to every $P_{x}$ except for a single center tangency, we get a surface isotopic to $Q_{b}$ and has at most one saddle tangency in $P \times[a-\epsilon, a+\epsilon]$. This means that after the isotopy, $Q_{b}$ is irreducible with respect to $P \times I$ and Theorem 1.1 follows from Lemma 2.2 and Lemma 3.3. So we may assume that $D_{\gamma} \cap F=\gamma$ for any component $\gamma$ of $\partial F$ that is trivial in $P_{a \pm \epsilon}$.

Let $\widehat{F}$ be the union of $F$ and all the disks $D_{\gamma}$ in $Q_{b}$ bounded by $\partial F$ as above. We may push all such disks $D_{\gamma}$ into $P \times(a-\epsilon, a+\epsilon)$ and isotope $\hat{F}$ into a surface properly embedded in $P \times[a-\epsilon, a+\epsilon]$. By the construction, $\partial \hat{F}$ is essential in $P_{a \pm \epsilon}$. So $\widehat{F}$ has no disk component. If $\widehat{F}$ consists of annuli, then since $\partial \widehat{F}$ is essential in $P_{a \pm \epsilon}$, each annulus is either vertical or $\partial$-parallel in $P \times[a-\epsilon, a+\epsilon]$. Thus, after some isotopy, $Q_{b}$ becomes irreducible with respect to $P \times I$ and Theorem 1.1 follows from Lemma 2.2 and Lemma 3.3. So we may assume $\chi(\widehat{F})$ is either -2 or -1 , ie at most one component of $\partial F$ is trivial in $P_{a \pm \epsilon}$.

Suppose $\chi(\hat{F})=-1$. If $\hat{F}$ is a once-punctured torus, then $\hat{F}$ must be incompressible in $P \times[a-\epsilon, a+\epsilon]$. Otherwise a compression on $\widehat{F}$ yields a disk, contradicting that $\partial \widehat{F}$ is essential in $P_{a \pm \epsilon}$. As $\widehat{F}$ is properly embedded in the product $P \times[a-\epsilon, a+\epsilon]$, $\widehat{F}$ must be $\partial$-compressible. A $\partial$-compression on $\widehat{F}$ yields an incompressible annulus with both boundary circles in $P_{a-\epsilon}$ (or $\left.P_{a+\epsilon}\right)$. So the resulting annulus is $\partial$-parallel. Since $\hat{F}$ is incompressible, this implies that $\hat{F}$ itself is $\partial$-parallel. Hence we can perform an isotopy on $\widehat{F}$ so that $Q_{b}$ becomes irreducible with respect to $P \times I$. 
Similarly, if $\widehat{F}$ is a pair of pants, then $\widehat{F}$ must be incompressible but $\partial$-compressible. So a $\partial$-compression on $\widehat{F}$ yields one or two incompressible annuli. This implies that either $\widehat{F}$ is $\partial$-parallel or we can perform an isotopy on $\widehat{F}$ so that $\widehat{F}$ is transverse to each $P_{x}$ except for a single saddle tangency. In either case, we can isotope $\widehat{F}$ so that $Q_{b}$ becomes irreducible with respect to $P \times I$ and Theorem 1.1 follows from Lemma 2.2 and Lemma 3.3 .

Therefore, we may assume $\chi(\widehat{F})=-2$. Hence $F=\widehat{F}$ and every component of $\partial F$ is essential in $P_{a \pm \epsilon}$.

Since $b$ is not labelled and since every component of $\partial F$ above is essential in $P_{a \pm \epsilon}$, at each regular level $x \in(0,1)$, if a component of $P_{x} \cap Q_{b}$ is trivial in $P_{x}$, then it must also be trivial in $Q_{b}$. Thus, we can apply Lemma 2.1 on $Q_{b} \cap(P \times([0, a-\epsilon] \cup[a+\epsilon, 1]))$. So after some isotopies, $Q_{b}$ satisfies all the conditions in Lemma 2.1 except for the level $P_{a}$ where $P_{a} \cap Q_{b}$ contains 2 saddle tangencies. Moreover, since $b$ is not labelled, $Q_{b} \cap \Sigma_{U} \neq \varnothing$ and $Q_{b} \cap \Sigma_{V} \neq \varnothing$. Hence $Q_{b} \cap P_{s} \neq \varnothing$ for every $s$.

Claim A Let $\sigma$ and $w$ be any components of $Q_{b} \cap P_{a-\epsilon}$ and $Q_{b} \cap P_{a+\epsilon}$ respectively. Then $d(\sigma, w) \leq 2=-\chi(F)=-\chi\left(Q_{b} \cap(P \times[a-\epsilon, a+\epsilon])\right)$.

Proof of Claim A If $\sigma$ is a boundary curve of a vertical annulus component of $Q_{b} \cap(P \times[a-\epsilon, a+\epsilon])$, then $\sigma$ is isotopic to a component of $Q \cap P_{a+\epsilon}$ and hence $d(\sigma, w) \leq 1$ for any curve $w$ in $Q \cap P_{a+\epsilon}$. So we may assume neither $\sigma$ nor $w$ is a boundary curve of a vertical annulus. Thus $\sigma$ and $w$ are both components of $\partial F$.

Let $\Omega$ be the union of the components of $P_{a} \cap Q_{b}$ that contain the 2 saddle tangent points. So $\Omega$ is a possibly disconnected graph with 2 vertices of valence 4 . Let $N(\Omega)$ be a regular neighborhood of $\Omega$ in $P_{a}$ and let $\pi: P \times I \rightarrow P_{a}$ be the projection, then $\pi(\partial F) \subset N(\Omega)$ after isotopy. Since $P$ has genus at least 2, there must be an essential curve $\alpha$ in $P_{a}$ disjoint from $N(\Omega)$. So $d(\sigma, w) \leq d(\sigma, \alpha)+d(\alpha, w) \leq 2=-\chi(F)$.

Now Theorem 1.1 follows from the argument in the proof of Lemma 2.2. As in the proof of Lemma 2.2, let $s_{0}<\cdots<s_{n}$ be a collection of regular levels such that $s_{0}=\delta$, $s_{n}=1-\delta$ for a small $\delta$ and there is exactly one critical level in each $P \times\left(s_{i}, s_{i+1}\right)$. Let $\Gamma_{i}=Q \cap P_{s_{i}}$ for each $i$.

Since we assume $Q$ is bicompressible in this section and since $M$ is irreducible, if $Q$ is a torus, then $M$ must be a lens space and $P$ and $Q$ must be isotopic Heegaard surfaces of the lens space (see Bonahon-Otal [2]). So we may assume $g(Q) \geq 2$.

Suppose $d(\mathcal{U}, \mathcal{V})>g(Q)$. Since $g(Q) \geq 2$, we have $d(\mathcal{U}, \mathcal{V})>4$. Let $k$ be the smallest integer such that $d\left(\mathcal{U}, \Gamma_{k}\right) \neq 0$ and $l$ the largest integer such that $d\left(\Gamma_{l}, \mathcal{V}\right) \neq 0$. By 
Claim A above and Claim 1 in the proof of Lemma 2.2, $d\left(\mathcal{U}, \Gamma_{k}\right)$ and $d\left(\Gamma_{l}, \mathcal{V}\right)$ are either 1 or 2 and $k \leq l$. Without loss of generality, we assume $k<l$.

Similar to the proof of Lemma 2.2, $\Gamma_{k}$ and $\Gamma_{l}$ must be essential in $Q_{b}$. Let $Q^{\prime}=Q_{b} \cap$ $\left(P \times\left[s_{k}, s_{l}\right]\right)$, and let $U^{\prime}$ and $V^{\prime}$ be the two components of $M-P \times\left(s_{k}, s_{l}\right)$ containing $G^{U}$ and $G^{V}$ respectively, $F_{U}=Q_{b} \cap U^{\prime}$ and $F_{V}=Q_{b} \cap V^{\prime}$. Since $\Gamma_{k}$ and $\Gamma_{l}$ are essential in $Q_{b}, F_{U}, Q^{\prime}$ and $F_{V}$ are essential subsurfaces of $Q_{b}=F_{U} \cup Q^{\prime} \cup F_{V}$.

Claim B Let $\sigma_{k}$ be any component of $\Gamma_{k}$, then $d\left(\sigma_{k}, \mathcal{U}\right) \leq 1-\chi\left(F_{U}\right)$.

Proof of Claim B If a component $A$ of $F_{U}$ is a $\partial$-parallel annulus in $U^{\prime}$, then we may first isotope $A$ into $P \times\left(s_{k}-\epsilon, s_{k}\right]$. Then we isotope $A$ so that $A$ is transverse to each $P_{x}$ except for a circle tangency. Since $\partial F_{U}$ is essential in $P_{s_{k}}$, after the isotopy, $Q_{b}$ still satisfies the conditions in Lemma 2.1 except at the level $P_{a}$ as above. Now we push $A$ out of $U^{\prime}$. After the isotopy, we still have $d\left(\mathcal{U}, \Gamma_{k}\right) \neq 0$. If $k$ is no longer the smallest number so that $d\left(\mathcal{U}, \Gamma_{k}\right) \neq 0$ after the isotopy, then we can find a new $k$ and proceed as above. Eventually $F_{U}$ does not contain any $\partial$-parallel annulus after some isotopies. We can view these isotopies as trivial isotopies, so by our assumptions above, $Q_{b} \cap \Sigma_{U} \neq \varnothing$ after the isotopies.

We first show that $d\left(\sigma_{k}, \mathcal{U}\right) \leq 2$. As in the proof of Lemma 2.2, $d\left(\sigma_{k}, \mathcal{U}\right) \leq 1$ if $k=0$. So we may assume $k>0$. By the definition of $k, d\left(\mathcal{U}, \Gamma_{k-1}\right)=0$. Thus there is a component $w$ of $\Gamma_{k-1}$ representing a vertex in $\mathcal{U}$. By Claim A above and the Claim 1 in the proof of Lemma 2.2, $d\left(\sigma_{k}, w\right) \leq 2$ and hence $d\left(\sigma_{k}, \mathcal{U}\right) \leq 2$.

Since $F_{U}$ is an essential subsurface of $Q_{b}, \chi\left(F_{U}\right) \leq 0$. Since $d\left(\sigma_{k}, \mathcal{U}\right) \leq 2$ and $\chi\left(F_{U}\right) \leq 0$, to prove the claim, we only need to consider the case that $\chi\left(F_{U}\right)=0$. Suppose $\chi\left(F_{U}\right)=0$. Since $d\left(\mathcal{U}, \Gamma_{k}\right) \neq 0, F_{U}$ consists of incompressible annuli in $U^{\prime}$. Let $A$ be the component of $F_{U}$ that contains $\sigma_{k}$. If $A$ is also $\partial$-incompressible, then $A$ can be isotoped away from any compressing disk of $U^{\prime}$ and hence $d\left(\sigma_{k}, \mathcal{U}\right) \leq$ $1=1-\chi\left(F_{U}\right)$. If $A$ is $\partial$-compressible, then since $F_{U}$ contains no $\partial$-parallel annulus, a $\partial$-compression on $A$ yields a compressing disk of $U^{\prime}$ disjoint from $A$. Thus, $d\left(\sigma_{k}, \mathcal{U}\right) \leq 1=1-\chi\left(F_{U}\right)$ in any case.

Similar to Claim B, for any component $\sigma_{l}$ of $\Gamma_{l}, d\left(\mathcal{V}, \sigma_{l}\right) \leq 1-\chi\left(F_{V}\right)$. Although $P_{a} \cap Q_{b}$ contains 2 saddle tangencies, by Claim A and our assumptions on $Q_{b}$, Claim 3 in the proof of Lemma 2.2 also holds in this case, ie there is a component $\sigma_{k}$ of $\Gamma_{k}$ and a component $\sigma_{l}$ of $\Gamma_{l}$ such that $d\left(\sigma_{k}, \sigma_{l}\right) \leq-\chi\left(Q^{\prime}\right)$.

Since $Q^{\prime}, F_{U}$ and $F_{V}$ are essential subsurfaces of $Q_{b}, d(\mathcal{U}, \mathcal{V}) \leq d\left(\mathcal{U}, \sigma_{k}\right)+$ $d\left(\sigma_{k}, \sigma_{l}\right)+d\left(\sigma_{l}, \mathcal{V}\right) \leq 1-\chi\left(F_{U}\right)-\chi\left(Q^{\prime}\right)+1-\chi\left(F_{V}\right)=2-\chi(Q)=2 g(Q)$. Thus Theorem 1.1 is proved. 


\section{References}

[1] D Bachman, S Schleimer, Surface bundles versus Heegaard splittings, Comm. Anal. Geom. 13 (2005) 903-928 MR2216145

[2] F Bonahon, J-P Otal, Scindements de Heegaard des espaces lenticulaires, Ann. Sci. École Norm. Sup. (4) 16 (1983) 451-466 (1984) MR740078

[3] A J Casson, C M Gordon, Reducing Heegaard splittings, Topology Appl. 27 (1987) 275-283 MR918537

[4] D Gabai, Foliations and the topology of 3-manifolds, J. Differential Geom. 18 (1983) 445-503 MR723813

[5] K Hartshorn, Heegaard splittings of Haken manifolds have bounded distance, Pacific J. Math. 204 (2002) 61-75 MR1905192

[6] W J Harvey, Boundary structure of the modular group, from: "Riemann surfaces and related topics: Proceedings of the 1978 Stony Brook Conference (State Univ. New York, Stony Brook, N.Y., 1978)", Ann. of Math. Stud. 97, Princeton Univ. Press, Princeton, N.J. (1981) 245-251 MR624817

[7] J Hempel, 3-manifolds as viewed from the curve complex, Topology 40 (2001) 631-657 MR1838999

[8] T Kobayashi, O Saeki, The Rubinstein-Scharlemann graphic of a 3-manifold as the discriminant set of a stable map, Pacific J. Math. 195 (2000) 101-156 MR1781617

[9] T Li, On the Heegaard splittings of amalgamated 3-manifolds Available at http:// www2.bc.edu/ taoli/amalg.pdf

[10] $\mathbf{T} \mathbf{L i}$, Images of the disk complex preprint

[11] H Rubinstein, M Scharlemann, Comparing Heegaard splittings of non-Haken 3manifolds, Topology 35 (1996) 1005-1026 MR1404921

[12] M Scharlemann, Local detection of strongly irreducible Heegaard splittings, Topology Appl. 90 (1998) 135-147 MR1648310

[13] M Scharlemann, A Thompson, Heegaard splittings of (surface) $\times I$ are standard, Math. Ann. 295 (1993) 549-564 MR1204837

[14] M Scharlemann, M Tomova, Alternate Heegaard genus bounds distance, Geom. Topol. 10 (2006) 593-617 MR2224466

[15] W P Thurston, Foliations of manifolds which are circle bundles, $\mathrm{PhD}$ thesis, University of California, Berkeley (1972)

Department of Mathematics, Boston College, Chestnut Hill, MA 02467, USA

taoli@bc.edu

http://www2.bc.edu/ taoli/

Received: 7 January 2007 Revised: 1 June 2007

Algebraic $8 \mathcal{G}$ Geometric Topology, Volume 7 (2007) 